\title{
Promoting Women to Editorial Leadership Positions at Academic Psychiatry
}

\author{
John Coverdale ${ }^{1} \cdot$ Rashi Aggarwal $^{2} \cdot$ Mary Morreale $^{3} \cdot$ Richard Balon $^{3} \cdot$ Eugene V. Beresin $^{4} \cdot$ Anthony P. S. Guerrero $^{5}$. \\ Alan Louie $^{6}$ - Adam Brenner ${ }^{7}$
}

Published online: 5 February 2021

(C) Academic Psychiatry 2021

Appointments to editorial boards require a respected history of productivity in writing and research. They also ordinarily require contributions to the topics of interest related to the mission of a journal and to the journal itself as a peer reviewer or an author. Appointments are made at the discretion of the editor in chief, with possible oversight by a governing board in some journals. Serving on an editorial board such as for the journal Academic Psychiatry is an honor and a mark of accomplishment in an academic career.

Is it justified to consider gender in appointments to journal leadership positions, including membership on the editorial board? It might be assumed that men would predominate on editorial boards for the reason that they are advantaged in their academic careers due to implicit and explicit biases. Women may be disadvantaged by factors such as child-rearing obligations; by possible biases that operate in their hiring and promotion, such as challenges in serving as leaders of maledominated specialties; and by a relative lack of mentors, sponsorship, and role models during their academic careers. Explicitly considering gender may force journal leaders to examine and question their implicit and explicit biases and may advance academic publishing to a time when gender no

John Coverdale

jhc@bcm.edu

1 Baylor College of Medicine, Houston, TX, USA

2 Rutgers New Jersey Medical School, Newark, NJ, USA

3 Wayne State University, Detroit, MI, USA

4 Harvard Medical School, Boston, MA, USA

5 University of Hawai'i John A. Burns School of Medicine, Honolulu, HI, USA

6 Stanford University, Stanford, CA, USA

7 University of Texas Southwestern Medical Center, Dallas, TX, USA longer needs to be considered in appointments because women receive the same opportunities as men.

Our goals for this editorial are to examine this question for the Academic Psychiatry journal. In doing so, we will consider possible gender disparities on editorial boards, including those of psychiatry journals. We will also discuss the potential advantages that women may bring to leadership positions on journals. Our intent is not to be all inclusive but to aim to justify an answer for how Academic Psychiatry might operate moving forward and to provide some transparency to our thinking on this topic of importance.

\section{Is There a Disparity of Gender on Editorial Boards?}

There is evidence indeed of a disparity of women on editorial boards in academic settings. One comprehensive study [1] aimed to quantify the percentage of women on editorial boards of psychiatry journals. This study was a search of 119 journals in the top tier of Clarivate Analytics' Journal Citation Reports (2017). In one phase of the study, Hafeez et al. extracted data on the 8423 editorial board members from the selected journals based on the seniority of their editorial or advisory role. A second phase collected more specific data, such as the $\mathrm{H}$-index and number of publications from a random selection of $10 \%$ of the original sample with equal numbers of men and women. In total, women represented $30.4 \%$ of all editorial board members, which was a similar proportion across all levels of seniority of journal leadership positions. In addition, the USA and the UK were the leading countries in terms of numbers of women in editorial leadership positions [1].

In the second phase of the study, although women had half the mean numbers of publications compared to men, they served journals with the same mean impact factors. The authors argued that while there was a gender disparity across journals, a gender bias did not exist in that the gender disparity 
on editorial boards was comparable to existing disparities in the upper ranks of academic psychiatry and research. In our view, though, even should disparities be comparable, this is not to say that a gender bias does not necessarily exist. Nevertheless, the authors recommended that gender disparity should be eliminated and that each gender should represent half of all psychiatry editorial boards [1]. The authors made several practical suggestions for how this might occur, such as by encouraging women early in their psychiatry careers to engage in editorial tasks and by creating workshops and seminars to encourage women to join editorial boards.

Monique Mun and Esther Akinyemi [2] wondered whether an increase in women entering psychiatry was reflected in the prevalence of women on editorial boards. They chose 12 psychiatry journals with high impact factors in 2017 and collected data from the journal websites and other sources. They found that women were underrepresented in editorial positions and that women comprised only $23 \%$ of total positions across the selected journals. They also argued that there should be an equal representation of women on the editorial boards of influential journals.

\section{Our Perspective}

Women on editorial boards serve as role models and can inspire others to greater heights. The Academic Psychiatry Editor Emeritus, Dr. Laura Weiss Roberts, wrote that the presence of great women in medicine made becoming an editor in chief seem possible to her [3]. She also expressed her hope that early career academic colleagues would envision themselves in influential roles, including as journal editors, even if they were not seeing someone who looks like them in the job already. Of course, this perspective should apply equally well to other underrepresented groups, including racial or ethnic minorities and people located in poorly resourced geographic areas.

Women as editors or editorial board members can also mentor female and male colleagues about strategies for success in writing and publishing. Their unique experiences and appreciation of behind-the-scenes editorial organizational processes can encourage and enlighten prospective authors. Formal sponsorship programs that match women with academic leaders, facilitate access to beneficial relationships, and institutionalize the value of equal opportunity can enhance mentorship [4]. Men in editorial leadership can also excel as allies and mentors of women, roles that may perhaps depend to an extent on men's understanding of the challenges that women disproportionally face in developing their careers [5]. In addition, the voices of both men and women are required to create a more humane, compassionate, and equitable culture across medicine [6].
Women in editorial positions may also assist in countering any potential blind spots in editorial decision making and bring a wider range of ideas and solutions to journals. Their perspective might differ from men's regarding topics of importance in academic psychiatry. They might endorse different thresholds for acceptance or rejection of articles of different types throughout the steps of the editorial decision-making processes. Perhaps they are less likely to undervalue the work of papers authored by women or to undervalue work on topics related to women's issues, should implicit biases be operating to the disadvantage of women. Their presence alone might encourage some authors to submit articles on women's issues within the domain of the mission of the journal on an assumption by authors that the journal will be more receptive to those articles. These are untested hypotheses for sure, but their potential value should not be discounted. At the same time, criteria for publication should remain consistent, use appropriate methodologies, and require excellence in writing and properly drawn conclusions.

Women are now in the majority of medical students [7], and many have equal, if not better, application credentials as their male counterparts. The mentoring noted earlier should begin at the outset of medical school. Many women come into medicine with aspirations for research and scholarship. Early provision of academic mentors, particularly women role models, sets the stage for advancement in the field and helps women learn the knowledge, skills, and attitudes needed for academic success and for developing the skills requisite to leadership at journals. We have seen in many areas of our field that early mentorship has a higher yield of success down the road.

Our mission and wish for promoting women to leadership positions in academic medicine requires a consideration of the overall climate and culture of medicine. Medical schools, hospitals, and national organizations should cultivate professional development programs, from medical school through residency and faculty positions, to promote the advancement of women physicians in a wide range of leadership positions. Women should be advised to vie for leadership positions in medicaland hospital-based research; to seek leadership positions on committees and task forces locally, regionally, and nationally; and to run for leadership positions in professional organizations. We hope that these broad efforts will also enable progress in women's leadership roles at journals, including Academic Psychiatry.

\section{Conclusions}

The answer to the question that we posed is a resounding yes; we believe that gender should be considered in appointments to journal leadership positions, including membership on editorial boards, and that we should all look for opportunities to promote women to editorial leadership roles. This position 
should at least apply to Academic Psychiatry, because we cannot directly speak for other journals. How much gender should be weighed in applications to leadership positions is a matter for further discussion. We appreciate that it is only one consideration among a range of commensurate factors.

Other journals are already leading the way. For example, journals such as Annals of Emergency Medicine [8] and Journal of Experimental Medicine [9] and dental journal editorial boards [10] are aiming to increase the representation of women. The Lancet Group [11] states on its website its commitment to gender equity and to increasing the representation of women from low- and middle-income countries among editorial advisers. They have also refreshed editorial advisory boards for gender parity [12]. Moreover, their editors will not serve on panels at a public conference or event that do not include women ("manels"), and they aim for at least 50\% female speakers at planned professional events [11]. The British Medical Journal published a view arguing that medical journals must address gender bias in academic medicine, including journal-level disparities in the number of editors [13], following a view that when women face barriers to publication and journal leadership, the pace of discovery slows and critical perspectives are lost.

The perspectives of women, given their immense importance and contributions to Academic Psychiatry, hugely enrich our own team. We hope to continue to promote women to our journal's leadership positions in order to improve our editorial functions and decision making. We encourage women to apply to our board and editorial positions when opportunities open. We are also committed to assist the academic careers of women in psychiatry and to support their promotion through the ranks. It is a privilege indeed to enjoy the input, leadership, and support of women at Academic Psychiatry, and we want to ensure that our leadership is well represented by them.

\section{Compliance with Ethical Standards}

Disclosures On behalf of all authors, the corresponding author states that there is no conflict of interest.

\section{References}

1. Hafeez DM, Waqas A, Majeed S, Naveed S, Afzal KI, Aftab Z, et al. Gender distribution in psychiatry journals' editorial boards worldwide. Compr Psychiatry. 2019;94:152119. https://doi.org/ 10.1016/j.comppsych.2019.152119.

2. Mun M, Akinyemi E. Representation on the editorial boards of academic psychiatry journals: the gender gap. Acad Psychiatry. 2020;44:506.

3. Roberts LW. Where are the women editors? Acad Psychiatry. 2014;38:391-3.

4. Gottlieb AS, Travis EL. Rationale and models for career advancement sponsorship in academic medicine: the time is here; the time is now. Acad Med. 2018;93:1620-3.

5. Bickel J. How men can excel as mentors of women. Acad Med. 2014;89:1100-2.

6. Flaherty SM, Misra M, Scott-Vernaglia SE, Taveras EM, Israel EJ. Psyche meets the gatekeepers: creating a more humane culture for women in medicine. Acad Med. 2019;94:1665-8.

7. Association of American Medical Colleges. 2019 fall applicant, matriculant, and enrollment data tables. December 2019. Available at https://www.aamc.org/media/38821/download. Last accessed 1/7/21.

8. Kaji AH, Meurer WJ, Napper T, Nigrovic LE, Mower WR, Schriger DL, et al. State of the journal: women first authors, peer reviewers, and editorial board members at Annals of Emergency Medicine. Ann Emerg Med. 2019;74:731-5.

9. Nathan C, Nussenzweig MC, Pulvirenti T. JEM advisory editorial board: increasing diversity. J Exp Med. 2017;214:2169.

10. Ioannidou E, Rosania A. Under-representation of women on dental journal editorial boards. PLoS One. 2015;10(1):e0116630. https:// doi.org/10.1371/journal.pone.0116630.

11. The Lancet Group. The Lancet Group's commitments to gender equity and diversity. Available at www.thelancet.com/diversity. Last accessed 04/30/2020.

12. Clark J, Horton R. What is the lancet doing about gender and diversity? Lancet. 2019;393:508-10.

13. Silver JK. Medical journals must tackle gender bias. BMJ. 2019;367:15888. https://doi.org/10.1136/bmj.15888.

Publisher's Note Springer Nature remains neutral with regard to jurisdictional claims in published maps and institutional affiliations. 